\title{
NEUERE FUTTERPFLANZEN IN DER DEUTSCHEN FUTTERPRODUKTION
}

\author{
A. STÄHLIN ${ }^{1}$ \\ Justus Liebig-Universität, Giessen, Deutschland
}

Im Jahre 1872 wurde eine Broschüre von William LöBE (28) geschrieben mit dem Titel "Landwirtschaftlicher Futterbau mit besonderer Rücksicht auf neue Futterpflanzen und Futtergemengen. Nach dem 90 Jahre alten Vorwort dieses Büchleins können auch die in den letzten Jahren und Jahrzehnten in Deutschland erprobten neuen Futterpflanzen klassifiziert werden in

1) solche, die sich wegen ihrer als wertvoll erwiesenen Eigenschaften das Bürgerrecht in der deutschen Landwirtschaft erworben haben,

2) in solche, deren Anbauwürdigkeit noch nicht genügend untersucht worden ist,

3) in solche Arten, bei denen es erwiesen ist, dass sie zum Anbau nicht tauglich sind, die aber als Objekte einer frechen Spekulation immer wieder mit marktschreierischer Propaganda auf alle mögliche Art angeboten werden.

Bei den neuen anbauwürdigen Arten handelt es sich zum Teil um altbekannte Nutzpflanzen, die infolge neuer Methoden der Züchtung, des Anbaues oder der Ernte ihr Areal seit dem Zweiten Weltkrieg haben ausdehnen können, zum Teil aber auch um echte Novitäten, wie die Gärtner ihre Blumen- und sonstigen neuen Pflanzenarten und -sorten bezeichnen. Während die meisten Arten der ersteren Gruppe seit langem zu den Arten mit vollem Bürgerrecht gehören, befinden sich unter den letzteren, den Novitäten, sowohl Arten, deren Wert oder Unwert für ganz Deutschland oder für grössere Landschaftsteile noch nicht feststeht, als auch Arten, von denen man, wie das 90 jährige Büchlein bereits sagt, befürchten muss, dass nur der Verkäufer des Saatgutes von ihrem Anbau profitiert.

$\mathrm{Zu}$ der ersten Gruppe, d.h. zu den Arten mit klarer Anerkennung ihres Anbauwertes, gehört der Mais $(31,38)$. Er kann in Deutschland auf eine schon mehr als 100 jährige Geschichte als Körner- und Futterpflanze zurückblicken. Wenn er trotz-

${ }^{1}$ Vortrag, gehalten am 19. 9. 63 vor der Finnischen Landwirtschaftswissenschaftlichen Gesellschaft in der Forstwirtschaftlichen Fakultät 
dem genannt wird, geschieht es mit vollem Recht, weil sein Anbau seit dem Zweiten Weltkrieg einen neuen starken Impuls durch die Einfuhr amerikanischer und die Züchtung europäischer Hybridsorten erfahren hat. Die klare Klassifizierung der FAO in Reifegruppen mit dreistelligen Zahlen für jede Sorte hat ebenfalls dazu beigetragen, dass der Wert einer Sorte für den einen oder anderen Nutzungszweck schnell erkannt werden kann. Die Deutsche Landwirtschafts-Gesellschaft hat diese internationalen Regeln für die deutsche Landwirtschaft mit geringfügigen, aber notwendigen Änderungen der Reifebezeichnungen zur Gruppierung der deutschen Sorten übernommen (Darst. 1). Je höher die erste Ziffer ist, desto später ist die Körnerreife der betreffenden Maissorte. Das amerikanische Maissortiment reicht, wenigstens theoretisch, von $100-999$, in Deutschland aber wird eine Sorte mit der FAO-Zahl über 350 nicht mehr reif.

Darste 11 u n 1

Gruppierung der deutschen Maissorten nach den FAO-Zahlen

FAO-Zahl
von 150 bis 199
,$\quad 200$ bis 249
, 250 bis 299
300 bis 349
350 und mehr

\author{
Reifegruppe \\ früh reifend \\ mittelfrüh reifend \\ mittelspät reifend \\ spät reifend \\ sehr spät reifend
}

In Deutschland werden 3 Nutzungsarten von Mais geübt, zur Körnergewinnung, zur Einsäuerung und zur Grünfütterung. Die Grünmaisernte kann vom Erscheinen der Rispe bis zur Milchreife der Körner erfolgen, Silomais aber soll die Teigreife erreicht haben, damit eine gute Silage gelingt, d.h. das Korn kann bei der Nagelprobe noch eingedrückt werden, ohne dass ein milchiger Saft austritt. Für die Körnergewinnung sollen wenigstens die erst angelegten Kolben die Gelbreife erreichen.

Während in Deutschland eine Zahl von 8-10 Pflanzen $/ \mathrm{m}^{2}$ für Körner- und bis 15 Pflanzen $/ \mathrm{m}^{2}$, je Sorte verschieden, für Silonutzung optimal ist, muss und darf Grünmais sehr viel enger, bis 50 und 60 Pflanzen $/ \mathrm{m}^{2}$, stehen. Diese dichtstehenden Grünfuttermaispflanzen bilden kaum mehr Kolben aus und diese Kolben sind schlecht befruchtet. Dafür steigt in den dünneren Stengeln der Zuckergehalt, weil die Maisstengel Reservestoffbehälter vor der Ausbildung der Körner sind. So beträgt im Grünfuttermais der Zuckergehalt mehr als $50 \%$ der Trockensubstanz gegenüber $40 \%$ bei einem Stand von 8 Pflanzen zur Silonutzung. Bereits beim Rispenschieben, d.h. etwa 10 Wochen nach der Saat, können weit über 10 Tonnen Trockenmasse geerntet werden, wobei wenig Unterschiede zwischen der mittelfrühen und der mittelspäten Reifegruppe bezüglich des Leistungszeitfaktors, d.h. des Zuwachses an Trockensubstanz in der Zeiteinheit, bestehen. Nur die späten Sorten kommen wegen langsamerer Entwicklung nicht mit. Es muss indes angesichts des grossen Saatgutbedarfs für Grünmais bis $100 \mathrm{~kg} / \mathrm{ha}$ und mehr eine Kalkulation 
aufgestellt werden, ob der niedrigere Preis für späte und sehr späte Sorten nicht den späteren Schnittzeitpunkt und den etwas kleineren Leistungszeitfaktor dieser Sorten wettmacht.

Nicht umsonst baut die bäuerliche Praxis der Bundesrepublik Deutschland im Durchschnitt der letzten 5 Jahre nur 6000 ha Körnermais (1962 allerdings 13000 ha) und 8-10000 ha Silomais an, aber 40-50000 ha für Grünmaisnutzung, weil dieser eine grosse Ertragssicherheit und damit eine Sicherung des Futterbedarfs bei Sommerstallhaltung gegenüber den meisten anderen Grünfutterpflanzen, besonders in trockenen Jahren aufweist. Selbstverständlich ist eine nicht vollkommene Zusammensetzung in der Grünmasse festzustellen, weil das Verhältnis von Eiweiss zu Kohlenhydraten sehr weit ist. Aber das gesamte Rindvieh frisst den Grünmais sehr gern, wobei kein Stengelteil übrig bleibt. Die Milchproduktion steigt und es wird dem Grünmais ein besonders angenehmer Geschmack des mit ihm erzeugten Fleisches nachgerühmt. Die lange Dauer der Nutzungmöglichkeit ohne grossen Qualitätsabfall - im Gegensatz zu der kurzen Nutzungsdauer von Grünroggen macht eine zeitliche Stufensaat von Grünfuttermais unnötig. Nur ein Schnitt vor der männlichen Blüte muss als Kindermord bezeichnet werden und wenn Mais erst nach der Milchreife geerntet wird, werden die Stengel nicht mehr restlos gefressen, weil der Ligningehalt in ihnen rasch zugenommen hat. Vielleicht muss bei der Sortenwahl für Grünfutterzwecke mehr als bisher auf die botanische Varietät geachtet werden, weil Zahnmaishybriden einen grösseren Ligningehalt als Hartmais aufweisen.

Der grosse Konkurrent des Grünmaises ist der Silomais, der noch mehr Nährstoffe, allerdings in längerer Zeit, von der Flächeneinheit bringt. Das soll zugegeben werden. Aber diese Mehrerträge werden, zum Teil durch die längere Wachstumszeit, zum Teil durch Einsäuerungsverluste, zum Teil finanziell durch die Kosten für die Silierung, aufgewogen, ganz abgesehen davon, dass in sehr vielen bäuerlichen Betrieben der eng zusammengebauten deutschen Dörfer kein Platz für die Aufstellung von Futtersilos ist. Schliesslich erreichen in ungünstigen Klimalagen und als Zweitfrucht selbst mittelfrühreifende Maissorten nicht in allen Jahren die Siloreife. Deswegen wird der Grünmais in Deutschland seinen Platz mit vollem Recht behaupten.

Vielleicht macht ihm in kühlen, regenreichen Lagen und auf saurem Boden der Hafer $(1,2,45)$ als Futterpflanze den Rang streitig. Sein Anbau als Körnerfrucht ist in der Bundesrepublik Deutschland in Parallele mit der Verringerung der Pferdezahl stark, wenn auch noch nicht ganz entsprechend, zurückgegangen, nämlich von 1,3 Millionen ha im Jahre 1950 auf 722000 ha im Jahre 1962. Dieser Rückgang ist aus Fruchtfolgegründen zu bedauern. Nach unserer Ansicht kann die Verwertung der Haferpflanze zur Grünmehlherstellung sowie als Grünfutter, Heu oder Silage einer weiteren Einschränkung der Haferanbaufläche entgegenwirken. Ausserdem eignet sich der Grünhafer ausgezeichnet als Glied in einem ganzjährigen Futterschlag; denn nach seiner Ernte, die in Deutschland Ende Mai bis Anfang Juli erfolgt, können Zweit- und Zwischenfrüchte in den bis zum Vegetationsende verbleibenden 3 Monaten noch einen vollen Ertrag bringen. 
Zur Futternutzung sind alle Hafersorten mit Ausnahme der extrem kurzstrohigen Mähdrescher-Hafer brauchbar. Eine Spezialzüchtung für Futterhafernutzung erscheint unnötig. Geradeso wie bei Mais dient der Haferhalm als Speicherorgan bis zur Milchreife für Zucker, bis dieser zum Aufbau des Stärkekörpers in das Korn abtransportiert wird. In Verbindung damit ist das Ergebnis der Ligninanalyse bedeutsam, da im Vergleich zu den Rohfaserprozenten eine fast scherenförmig entgegengesetzte Kurve, sogar in der Gesamtpflanze zu verzeichnen ist, d.h. vor der Milchreife ein deutlicher Abfall und dann ein kräftiger Anstieg der Ligninkurve. Die Rohfaserkurve aber fiel bereits vom Stadium der Blüte an sehr viel stärker ab, um erst nach der Milchreife steil anzusteigen. Dieser nicht parallele Verlauf der Rohfaser- und der Ligninkurve zeigt einerseits den stark komplexen Charakter der Rohfaser auf, andererseits dass die Aussagekraft der Rohfaserprozente für den Futterwert eines Rauhfutters sehr begrenzt ist, eben weil es auf die Zusammensetzung der Rohfaser ankommt. Auf jeden Fall ist dem Grünhafer eine grössere Zukunft in der Bundesrepublik zu prophezeien.

Dagegen ist an eine stärkere Ausweitung des Areals von Lolium multiflorum italicum $(6,16,39)$, der zweijährigen Form des Italienischen Raygrases, nicht zu denken, weil die Form nicht absolut winterhart ist. Vielleicht hat mehr Aussicht auf eine grössere Verbreitung die Subspecies gaudini dieser Art, d.h. die sommerannuelle Form, bei der wie bei der überjährigen mehrere Schnitte, aber ohne das Winterrisiko möglich sind. Neuere Züchtungen von gaudini sind auch nicht mehr rein generativ, sondern bilden viele wertvolle Blattmasse aus.

Eine Parallele zu diesen wertvolleren Formen von Lolium multiflorum hat man in den letzten Jahren bei der Züchtung von Sommerraps, Brassica napus var. arvensis f. anmua, beobachten können. Auch hier sind die neuen Zuchtsorten sehr viel blattreicher und wüchsiger als die alten, mehr generativen Sorten und schiessen deswegen nicht mehr so rasch in Blüte. Aber anscheinend erwächst dem Sommerraps in Gestalt von Brassica nigra, dem Schwarzen Senf, der in Giessen gezüchtet wird, ein starker Konkurrent. Dieser verdient Beachtung auch als Futterpflanze im Hinblick auf seine Frohwüchsigkeit und die Erntemenge im Zweitfruchtbau, sofern die überaus starke Ausfallneigung der Samen und der hohe Senfölgehalt der ganzen Pflanze durch Züchtung beseitigt werden können. Allerdings ruft bei der Verfütterung von allen Kreuzblütlern, Cruciferae, ein Gehalt an flüchtigen Senfölen in einer Höhe von $0,02 \%$ in der Trockensubstanz Störungen im tierischen Organismus hervor und die Qualität der Milch kann, wenn die Verfütterung während des Melkens geschieht, bis zu $60 \%$ der Milchproben fehlerhaft werden. Wegen seines hohen Senfölgehaltes ist der Schwarze Senf deshalb in die zweite Gruppe der neueren Futterpflanzen zu stellen, zu den Arten, deren Anbauwürdigkeit in der mitteleuropäischen Landwirtschaft erst genau geprüft werden muss, auch in ausgedehnten Fütterungsversuchen hinsichtlich Aufnahmewilligkeit und Wirkung.

Im Gegensatz zum Schwarzen Senf hat der Ölrettich, Raphanus sativus oleiformis $=$ chinensis, bereits Heimatrecht als Futterpflanze in Deutschland erworben, nachdem die Art in Giessen auf grössere Lagerfestigkeit gezüchtet worden ist. Vielleicht ist das Kraut von Ölrettich nicht so eiweissreich wie das von Gelbsenf, Sinapis alba, mit dem der Ölrettich in Anbau und Saatmenge, in Kälteresistenz 
und Wuchsschnelligkeit sehr vieles gemeinsam hat. Aber das Kraut von Ölrettich wird nicht so rasch überständig wie das von Gelbsenf und enthält noch in der Vollblüte bedeutend weniger Senföl, so dass nach der Verfütterung von Ölrettich keine Klagen über Gesundheitsschädigung der Tiere oder Minderung der Milchqualität bekannt geworden sind. Vielmehr wird angegeben, dass die Milchsekretion angeregt worden sei und die Qualität von Milch und Butter sich gebessert habe. Allerdings hätten sich manche Kühe an den Geschmack gewöhnen müssen. Der Senfölgehalt von Ölrettich nimmt erst stark zu, wenn die Samen voll entwickelt sind. Für die Beliebtheit dieser aufstrebenden Futterpflanze spricht eine Fläche von ca. 200 ha zur Saatgutvermehrung gegenüber ca. 500 ha von Winterraps und 1700 ha Sommerraps sowie 300 ha Winterrübsen.

Aus dem Grunde eines höheren Senfölgehaltes ist die alte Kulturpflanze Stoppel- oder Wasserrübe, Brassica rapa rapifera $(5,21,43)$, in einem Atemzug mit den Blattfutterpflanzen unter den Kreuzblütlern zu nennen. Ihr Anbau konzentriert sich in Mitteleuropa auf die leichten, etwas humushaltigen Böden, weil der Ertrag auf besseren Bodenarten eher ab- als zunimmt, infolge Verdichtung der Krume und Verunkrautung des Feldes; auch der Senfölgehalt wird höher. Neben ihrer Genügsamkeit ist der Hauptvorteil der Wasserrübe in Mitteleuropa ihre erstaunlich kurze Vegetationszeit von nur 6-10 Wochen im Spätsommer und Herbst, ihr Hauptnachteil ist neben ihrer schlechten Haltbarkeit der hohe Wassergehalt - sie führt mit ca. $90 \%$ Wasser in der Rübe ihren Namen wirklich zu Recht. Ausserdem äussert sie einen sehr ungünstigen Einfluss auf die Milchqualität, schlechter als alle anderen Zwischenfrüchte. Wegen der schlechten Haltbarkeit wird sie nämlich in den Hauptanbaugebieten Nordwestdeutschlands möglichst rasch verbraucht, d.h. es werden bis $75 \mathrm{~kg}$ je Kuh und Tag verfüttert. Durch solche Mengen wird nicht nur der Geschmack von Milch und Butter rübenartig, scharf und sauer bis bitter, ohne dass die Aromastoffe bisher erfasst worden sind, sondern es wird auch die Gesundheit der Tiere gefährdet und geschädigt mit den Symptomen, wie sie von Senföl bekannt sind, als da sind Speichelfluss, Fieber, Kolik, Durchfall bis Darmkatarrh und stärkere Entzündungen der Schleimhäute in Magen und Darm.

Nun sind erstens neue Sorten in den Niederlanden und in Deutschland gezüchtet worden mit weniger Senföl in der Frischsubstanz der ganzen Pflanze, 0,008 \% statt $0,012 \%$, und mit einem höheren Anteil von mindestens $60 \%$ statt $40-45 \%$ Blatt. Das Blatt enthält mehr Nährstoffe als die Rübe, vor allem mehr verdauliches Rohprotein, $17,5 \%$ statt $10,3 \%$ in der Trockensubstanz.

Der zweite Grund für die Ausdehnung des Anbauareals der Wasserrübe als einer nunmehr wieder neu interessanten, weil mit weniger Fehlern behafteten Futterpflanze ist arbeits- und betriebswirtschaftlicher Natur. Es ist nämlich eine neue Erntemaschine entwickelt worden, die das Blatt mit 2 Gummibändern oder mit Greifern erfasst und so die Rübe aus der Erde zieht. Auch dafür ist ein reicherer Blattschopf von Vorteil, wie er den neuen Sorten angezüchtet worden ist. Diese Sorten vom Weseler-Typ mit den holländischen Sorten Gelria und Siga haben auch ein nur geschlitztes bis ganzrandiges und damit festeres Blatt an Stelle des stark gegliederten Blattes der alten Sorten. Das bedeutet eine grössere Frosthärte, die für die maschinelle Rodung nach einem Frühfrost sehr wichtig ist. Damit die 
Rüben leicht aus dem Boden zu ziehen sind und das Blatt bei hand- und maschinellem Roden nicht abreisst, muss jetzt auf die alte Rübenform der krummen Ochsenhörner und langen Pfahlwurzeln zugunsten von halblangen, stumpfen Rüben verzichtet werden. Der Ertrag an Rübe und Blatt ist deswegen bei den neuen Züchtungen nicht geringer geworden. Selbstverständlich darf für die maschinelle Rodung die Wasserrübe nicht mehr breitwürfig gesät werden, sondern sie muss in Reihen gedrillt werden, und zwar möglichst dünn, nur mit ca. $1 \mathrm{~kg} / \mathrm{ha}$. Jetzt wird die Rübe stärker als bisher eingesäuert, weil die neue Maschine Hilfestellung für eine rasche Füllung des Silos leistet. Hier werden die Senföle zu einem grossen Teil, bis $75 \%$, abgebaut und die Trockensubstanz wird um mindestens die Hälfte der in der Frischsubstanz befindlichen auf durchschnittlich $17 \%$ erhöht.

Einen ähnlichen Aufschwung hat in verschiedenen Gegenden von Deutschland der in Finnland ebenfalls seit langem angebaute Markstammkohl, Brassica oleracea acephala $(15,18,32)$, genommen, nicht so sehr wegen der Anwendung von Pflanzmaschinen, sondern vielmehr deswegen, weil sich die Züchtung intensiver als bisher mit dieser formenreichen Pflanze beschäftigt hat. Dabei sind 2 Sortengruppen gezüchtet worden, die sich in ihrem Futterwert und ihrer Silierbarkeit stark unterscheiden, der Blatt- oder Futterkohl, bei dessen Sorten der Blattanteil mengenmässig überwiegt, und der eigentliche Markstamm- oder Strunkkohl, dessen Ertrag höher ist und bei dem der Stengel das meiste dazu beiträgt. Schon früher bestanden grosse Unterschiede im Futterwert der Sorten von der letzteren Gruppe zwischen dem weniger verholzenden Dickstrunk und Sorten mit Spazierstöcken als Stengeln, aber auch zwischen den wertvolleren grün- und den weniger gehaltreichen blaustengeligen Sorten. Zur Erhöhung der Erntemasse dürfte die Züchtung von Kompromiss-Sorten mit dickem Stengel, aber vielen Blättern das Optimum darstellen. Dann wird der Markstammkohl weiter an Boden gewinnen, sowohl als Haupt- als auch als Zwischenfruchtfutterpflanze zur Verlängerung der Frischfutterperiode bis etwa Weihnachten, gerade weil sein Senfölgehalt wegen seiner Zugehörigkeit zur Gattung Kohl gering ist und nur geringe Bedenken gegen eine Massenverfütterung von Markstammkohl wegen des Auftretens von Kohlanämie bestehen.

Bisher wurde nur über alte und wieder neue Futterpflanzen berichtet, die aus irgendeinem Grund, vor allem weil umgezüchtet, in Mitteleuropa als neu und beaohtenswert ermittelt worden sind. Eine Art aber ist noch vor 20 Jahren in Deutschland überhaupt nicht angebaut worden. Es handelt sich um den Alexandriner Klee, Trifolium alexandrinum $(9,12,13,25,27,34,39,40,41)$, der seit den 50er Jahren sehr viel Redens von sich gemacht hat. Er war plötzlich da, so dass man von ihm, wie von Caesar, sagen kann: veni - vidi - vici $=$ er kam, sah, siegte. Geradeso als ob die Zeit noch nicht für ihn reif gewesen sei, hat ein zweimaliger Versuchsanbau in Mittel- und Westeuropa keine Folgen für die Praxis gehabt. Im Jahre 1927 hat nämlich ein Anbauversuch bei München mit guten Erfolg stattgefunden und ebenso hat bereits 1854, also vor mehr als 100 Jahren, die Holländische Gesandtschaft in Kairo Saatgut nach Hause geschickt, das mit bestem Erfolg angebaut worden ist. Aber weil beide Male kein Saatgut gewonnen werden konnte, schliefen Anbau und Erkenntnis sofort wieder ein. Jetzt hat sich Alexandriner Klee mit 2-5 Schnitten, soviel also wie Luzerne, Medicago sativa, in Mitteleuropa und mit beachtlichen 
Erträgen als Ersatzfutterpflanze, aber auch als Sommerklee und Hauptfrucht mit Lolium multiflorum gaudini, Bürgerrecht in Deutschland erworben.

Die Feststellung des grossen Anbauwertes von Alexandriner Klee gilt indes nur für die italienischen, nicht für die portugiesischen und jugoslawischen oder amerikanischen Herkünfte, sowie nicht für die meisten ägyptischen Sorten. Von diesen sind einige nur einmal zu ernten wie eine nahverwandte Art, der Palermo-Klee oder Sparrige Klee, Trifolium squarrosum, der von unreellen Firmen gelegentlich nach Deutschland importiert wird, wegen seines gröberen Korns Käufer findet und nach einem befriedigenden Schnitt wegen mangelnden Nachwuchses gänzlich versagt. Zum Glück ist er mit seinem grösseren Tausendkorngewicht, den längeren Nebenblattzähnen und den sparrig abstehenden Kelchblättern leicht vom Alexandriner Klee zu unterscheiden. Während also Trifolium squarrosum $\mathrm{zu}$ der anfangs genannten Gruppe III der Futterpflanzen gehört, an deren Verkauf nur der unkönigliche, marktschreierische Händler profitiert, sind die guten Sorten und Herkünfte von Trifolium alexandrinum es wert, angebaut zu werden. Wohl kann seine Futterqualität nicht an die von Rotklee heranreichen, weil der Wassergehalt höher ist, aber er wird relativ gern gefressen.

Die Beimengung von Einjährigem Raygras erhöht den Mengenertrag, ohne dass das Eiweiss-Stärkeverhältnis allzu weit wird. Dabei interessiert der Vergleich von Alexandriner Klee mit der italienischen Herkunft von Rotklee. Diese kann, als Untersaat im Frühjahr in den Boden gebracht, mit raschem Wuchs nach der Getreideernte noch einen Schnitt im gleichen Jahr bringen, während der Alexandriner Klee, wegen höherer Lichtansprüche in der Jugend, als Untersaat weniger tauglich ist. Im Mengenvergleich aber schneidet der Alexandriner Klee bei Hauptanbau, mit Ausnahme von kühleren Lagen, bedeutend besser ab.

In der ersten Begeisterung wurde er oft dort angebaut, wohin er wegen der Ungunst der klimatischen Verhältnisse als wärmeliebende Pflanze des östlichen Mittelmeergebietes nicht gehört. Sein Areal in Mitteleuropa muss erst noch nach Versuchen abgesteckt werden, weil er gegen nasse und kalte Witterung äusserst empfindlich ist und nach deren Schock rotbraun wird, wobei er sich nur langsam erholt. Wie bei allen anderen Nutzarten ist eben die Wirkung von neuen Kulturarten nicht zu vergessen, damit die Gunst des Standortes richtig ausgewählt wird. Habituell ist der Alexandriner Klee der Luzerne sehr ähnlich. Aber er hat gelblichweisse, ziemlich kugelrunde Köpfchen, wie Trifolium repens oder noch mehr wie Trifolium montanum, und an ihnen kann er sicher erkannt werden, sobald er blüht.

Im Zusammenhang mit dem Alexandriner Klee ist als eine neue Art der zweiten Gruppe der Persische oder Wendeblumenklee, persisch Schabdar, Trifolium resupinatum, zu nennen. Dieser wird von Irak bis Ceylon mit dem Hauptgewicht im Iran, und dort in Höhen von 1000 bis $2800 \mathrm{~m}$ bei $600-800 \mathrm{~mm}$ Winterniederschlägen als Futterpflanze angebaut oder beweidet. In Westdeutschland hat er im Jahre 19625 Massenschnitte geliefert. Im blütenlosen Zustand sieht er wie ein grossblättriger Alsikeklee, Trifolium hybridum, aus, während er mit seinen kleinen rosafarbenen bis purpurvioletten Köpfchen keiner mitteleuropäischen Kleeart gleicht. Trifolium resupinatum gefällt im Vergleichsanbau mit Trifolium alexandrinum durch grössere 
Erträge an Grünmasse mit unbehaarten Blättern und durch eine grössere Kältehärte. Wir kennen von ihm 3 Typen, eine frühblühende und rein generative Form, die in Portugal Interesse gefunden hat und von der es im Iran auch einschnittige ohne Nachtrieb gibt, eine kriechende Form, die aus dem Süden Irans in USA als salzverträgliche und extensive Weidepflanze interessant geworden ist, und eine rosettenbildende Form, die in ihrer iranischen Heimat mit 7 Schnitten und einer Samenernte am meisten verbreitet ist. Sicher kann aus diesen verschiedenen Formen noch viel gezüchtet werden, und das muss geschehen, um für Mitteleuropa eine Kombination der massenbringenden Rosettenform mit raschem Jugendwachstum, eine gute Nachwuchsfreudigkeit und Trockenresistenz mit photoperiodisch neutraler Reaktion zu besserem Samenansatz zu schaffen. Trotz aller Schwierigkeiten, die bislang vor allem bei der Samenvermehrung bestehen, ist die Züchtung von Trifolium resupinatum in Deutschland bereits aufgenommen worden, in der Erwartung, dass diese Art den Alexandriner Klee wegen besserer Eigenschaften verdrängen werde.

In Finnland spielt, wie in ganz Skandinavien, die künstlich tetraploidisierte Form des Ackerrotklees, Trifolium pratense sativum, als Tetraklee eine grössere Rolle. In Deutschland wurde diese Zuchtmethode bisher wenig angewendet. Nun aber interessieren Sorten, die aus der Gruppe Frühklee tetraploidisiert worden sind, damit sie womöglich zu mehrjähriger Kleegrasnutzung in den deutschen Mittelgebirgen eingesetzt werden, die in der EWG existenzgefährdet sind.

Wie die Wasserrübe ist der Weisse Steinklee, Melilotus albus $(30,37)$, wegen neuer, erfolgversprechender Züchtungsversuche zu nennen. Es sieht nach diesen in Göttingen vorgenommenen Züchtungen so aus, als ob die beiden negativen Haupteigenschaften, die den Anbau von Weissem Steinklee in Mitteleuropa bisher uninteressant gemacht haben, endlich weggezüchtet worden seien, nämlich der Cumaringehalt, der von Blühbeginn an, mit etwa $1 \%$ in der Trockensubstanz, das Rindvieh an der Aufnahme grösserer Futtermengen gehindert hat, und die Grobstengeligkeit des meist einzigen Triebes, der bei den gewöhnlichen Formen blattarm ist, $2 \mathrm{~cm}$ dick wird und bald stark verholzt. Nunmehr ist es in Göttingen gelungen, luzerneähnliche Büsche mit gutem Blatt-Stengel-Verhältnis, niedrigerem Wassergehalt und einem nicht mehr störenden Gehalt an Cumarin durch Kreuzungs- und Auslesezüchtung zu schaffen. Das wäre ein grosser Gewinn, weil Melilotus albus noch mit Rohböden und harten Störschichten im Boden fertig wird und auch sonst anspruchslos und wetterhart ist. In Amerika sagt die Propaganda von ihm, dass er unfruchtbares Land bebauungsfähig und gutes Land besser mache. Daran ist sicher etwas wahr. Aber wir müssen noch abwarten, ob mit der Wegzüchtung des einzigen kräftigen Stengels nicht auch die starke Pfahlwurzel zur Durchdringung von ungünstigen Bodenschichten verschwunden ist. Überhaupt müssen wir es noch der Zukunft überlassen, ob die Praxis einen grossen Vorteil aus der jahrelangen Göttinger Arbeit, die schöne wissenschaftliche Ergebnisse gezeitigt hat, ziehen kann. Der Steinklee ist also eine ausgesprochene Pflanze der zweiten Kategorie, bei der Untersuchungen über Eignung und Wert angestellt werden müssen.

Das gleiche ist bei den 3 Lathyrus-Arten der Fall, von denen 2, nämlich Lathyrus sativus und cicera $(20,26,35)$, in Giessen züchterisch bearbeitet werden. Beide 
Arten stammen aus dem Mittelmeergebiet und verlangen deshalb mehr Wärme als sie in den meisten Lagen und Jahren in Mitteleuropa finden. Aus diesem Grunde ist auch die eine, die Rote Platterbse, Lathyrus cicera, nur als Gründüngungspflanze im Weinklima und zur Bodenbedeckung in Weinbergen vorgeschlagen worden. Die Weinbauern der Champagne haben für sie Interesse gezeigt. Bisher hat keine unreelle Samenfirma Propaganda für sie gemacht, sonst wäre die Art in die dritte Gruppe, in die der nicht anbauwürdigen Futterpflanzen, zu stellen.

Viel mehr dürfte auch die Saatplatterbse, Lathyrus sativus, nicht wert sein, selbst nicht in der weissamigen Form, die das Alkaloid Lathyrin in ungefährlichen Mengen enthält, so dass die Tiere, vor allem Pferde, nicht an Lathyrismus erkranken, einer Krankheit, die sich in Atembeschwerden und Lähmungserscheinungen, schliesslich in Tod durch Erstickung äussert. Abgesehen von dieser Gefahr stellt die Saatplatterbse, die übrigens eine so uralte Kulturpflanze ist, dass ihre Stammform, vielleicht die Rote Platterbse, nicht feststeht, sehr hohe Ansprüche an die Wärme des Standorts, so dass sie nur im warmen Weinklima angebaut werden kann. Da sie zudem auf demselben Boden wie die gewöhnliche Erbse, Pisum sativum, am besten wächst, also einem kalkreichen, etwas bindigen Boden von mittlerer Feuchtigkeit, und weil die Erbse ausserhalb des Weinklimas immer, sowohl bei Futter- als auch bei Körnernutzung, besser abschneidet, ist wohl auf die Züchtung von Lathyrus sativus im gemässigten Klima Mitteleuropas ganz zu verzichten und Anbau und Züchtung der dürreverträglichen, eiweissreichen und selbstverträglichen Art den Mittelmeerländern ganz zu überlassen, wo sie seit dem Altertum angebaut wird. Immerhin ist eine in Giessen gezüchtete Sorte in Deutschland zugelassen (26).

Vielleicht sollte die Tanger-Platterbse, Lathyrus tingitanus, mehr Beachtung finden. Sie ist mehr massen- und hochwüchsig als die Saatplatterbse und erst recht als die Rote Platterbse. Die Art eignet sich deshalb gut als Gemengeanteil mit Hafer und anderen Futterleguminosen. Ihr Kraut verholzt noch etwas später als das der Saatplatterbse und kann deshalb bis zu dem späten Zeitpunkt, wenn die Hauptmasse der Infloreszenzen bereits Hülsen angesetzt hat, noch grün verfüttert werden. Von Lathyrus tingitanus ist auch eine Erkrankung, wie von den dunkelsamigen Formen der Saatplatterbse, nicht bekannt geworden. Wahrscheinlich muss man sagen, dass die Art in der breiten Landwirtschaft Mitteleuropas weniger bekannt ist als ihr Futterwert es verdient. Die Kalkulation, ob Saatgut dieser Art und von anderen Leguminosen oder Stickstoffdünger beim Grün- und Silohaferanbau zu kaufen sei, ist, wegen der Bereicherung der Aminosäuren-Speisekarte für das Rindvieh durch das wertvollere Leguminoseneiweiss, sehr schwer aufzustellen (20).

Von der Gattung Lupinus $(11,42)$ kennen wir zwei Genzentren, in der Alten Welt das Mittelmeergebiet mit höchstens 11 nur einjährigen Arten, darunter mit den 3 für die mitteleuropäische Landwirtschaft relativ wichtigen Arten der Gelben, Blauen und Weissen Lupine, Lupinus luteus, angustifolius und albus, und in der Neuen Welt ein riesiges Gebiet von Nord- bis Südamerika mit sehr zahlreichen einjährigen und ausdauernden Arten, aber mit nur einer Art, die als halbe Kulturart in Mitteleuropa verwendet wird, nämlich mit der Vielblättrigen Lupine, Lupinus polyphyllus, die, nur vermehrt und bisher nicht gezüchtet, im Forst zur Stickstoffsammlung und Bodenverbesserung breitwürfig ausgesät wird. Die Indianer Perus 
haben eine andere Art, die wir nur als Zierpflanze kennen, die Verschiedenfarbige Lupine, Lupinus mutabilis, angebaut; die bitteren Samen, mit einem Tausendkorngewicht von ca. $150 \mathrm{~g}$ wie von L. angustifolius, wurden durch Wässern entbittert.

Unter den 460 Arten des südamerikanischen Subkontinents, unter den mindestens 100 Arten Mittelamerikas und den ebenso vielen Arten Nordamerikas befinden sich zahlreiche kulturwürdige Arten mit hohem Rohprotein- und Rohfettgehalt, mit hohem und mit niedrigem Tausendkorngewicht, mit hoher Grünmassenproduktion und mit der Eignung für das mitteleuropäische Klima. Nach dem Gesetz der Parallelvariationen können, wie bei L. luteus und angustifolius, Stämme mit geringem Alkaloidgehalt und mit geringer Platzneigung der Hülsen erwartet werden; sie sind von der mittelamerikanischen kleinkörnigen Ausdauernden Lupine, Lupinus perennis, und der genannten westamerikanischen Waldkulturpflanze, L. polyphyllus, bereits bekannt (11).

Das alles ist indes Zukunftsmusik; denn der Weg von der Wild- zur Kulturpflanze ist lang und für den Züchter dornenreich. Das zeigt die Geschichte von L. luteus und angustifolius, den schon im Altertum neben L. albus bekannten, Mittelmeerarten, die damals und bis in die 30er Jahre nur als Gründüngung verwendet werden konnten. Erst nach der Züchtung auf Bitterstoffarmut waren sie auch als Futterpflanzen und Körnerfrüchte brauchbar. Zur Zeit der deutschen Autarkiebestrebungen vor dem letzten Weltkrieg hat ihr Anbau einen grossen Aufschwung genommen, auch in Verdrängung der auf Sandboden zur Gründüngung wertvollen Bitterlupinen. Aber jetzt können ihre Sorten nicht leben und nicht sterben, weil das Eiweiss aus ihrem Anbau teurer erzeugt wird als der Weltmarkt es liefert.

Mit der Weissen Lupine, die bereits bei den Römern für die menschliche Ernährung gedient hat, ist, wie zu vielen anderen nicht sehr aussichtsreichen Züchtungen, die ernste, bis in den kulturellen Bereich gehende Frage zu stellen: Soll man die Liste der Kulturarten, deren Züchtung bis in das graue Altertum zurückreicht, verarmen lassen, muss man es tun oder haben wenigstens nicht staatliche Stellen die Aufgabe der Erhaltung für einen späteren eventuellen Bedarf? Diese Frage nach der Daseinsberechtigung alter Kulturarten und ihrer Züchtung steht in Gegensatz zu dem Thema über neue in Kultur genommene Arten. Aber es gehört doch für alle Kulturländer eng dazu, weil bei der Züchtung von alten und von neuen Arten durch moderne Methoden wertvolle Formen gewonnen werden können oder weil durch Aufgabe dieser Arten die Zuchtformen für immer von dieser Erde verschwinden würden.

Das Giessener Institut für Pflanzenbau und Pflanzenzüchtung hat als deutsche Zuchtstätte für landwirtschaftliche Sonderkulturen 2 weitere Arten in intensive Züchtung genommen und Sorten davon bis zur Zulassung gebracht. Es sind dies die Sonnenblume, Helianthus annuus $(3,4,33)$, und der Ölkürbis, Cucurbita pepo styriaca $(10,36)$. Bei beiden Arten könnte man sich in oberflächlicher Betrachtung streiten, ob sie mehr Futterpflanzen oder mehr Ölfrüchte sind. Aber wenn man sie näher betrachtet und ihre Vor- und Nachteile für die eine oder andere Nutzung abwägt, überwiegt bei beiden die Futternutzung wenigstens in Mitteleuropa. Solange bei der Sonnenblume das technische Problem der Trennung von Schale und ölreichem Kern nicht gelöst ist, so lange ist die Bedeutung der Sonnenblume als 
Futterpflanze in Mitteleuropa ungleich grösser denn als Ölfrucht, weil die Rückstände der Ölgewinnung bisher zu schalenreich und damit in Futterwert und Rentabilität nicht konkurrenzfähig sind. Ebenso ist die maschinelle Gewinnung der ölreichen Kürbiskerne aus den wasserreichen Kürbisfrüchten noch ein Problem.

Die Sonnenblume liefert zwar kein sehr hochwertiges und auch kein in grünem Zustand gern gefressenes Futter. Aber nach Giessener Züchtungsversuchen gibt es Formen mit weichen Haaren, so dass der Grund zur Ablehnung des frischen Sonnenblumenkrautes durch das Rindvieh wegfallen würde. Als stets gut gelingendes Gärfutter werden die Futtermassen der milchreif gewordenen Sonnenblume, die grösser oder so gross sind wie bei jeder anderen Zwischenfruchtfutterpflanze, anstandslos gefressen. In Giessen ist es auch gelungen, eine mittelspätreifende, standfeste und kürzerstengelige Sorte auf den Markt zu bringen. Sie wird sicher im mitteleuropäischen Klima reif, bildet mehr Masse im Haupt- und Stoppelfruchtbau als Sommerraps und eignet sich als Stützpflanze für Leguminosen und als Spätsaat bis Mitte August in gleicher Weise wie die alten Sorten, ohne dass die Genügsamkeit gegen Bodenarmut an Wasser und Nährstoffen, wie bei der Züchtung der Süsslupinen, verloren gegangen wäre. So ist die Sonnenblume in den neuen Giessener Sorten als eine sichere Ersatzpflanze für Mais in ungünstigeren Lagen zu bezeichnen, ebenfalls mit $100 \mathrm{t} /$ ha Grünmasse.

In Giessen ist es auch bei Ölkürbis gelungen, in einer rankenlosen, früher reifenden Sorte eine wertvolle Form zu züchten, die, allerdings nur im Weinklima, als Futterpflanze ihre Berechtigung hat. Bisher wurde Ölkürbis lediglich am warmen Fuss der Alpen in der Steiermark angebaut. Die Art ist eine subtropische Pflanze aus Mittelamerika und kann mit ihren Wärmeansprüchen ihre Herkunft nicht verleugnen. Wegen der Unsicherheit des Gelingens bei ungünstiger, nasskalter Sommerwitterung hat der feldmässige Anbau von Kürbis zu Futterzwecken in Mitteleuropa, im Gegensatz zu Ungarn, bisher wenig Verbreitung gefunden. Das ist zu bedauern, weil ein Kürbisfeld mit einem Ertrag von sehr oft mehr als 100 t/ha Ölkürbis und in Ungarn von ca. 200 t/ha Riesenkürbis trotz dem hohen Wassergehalt gut das Doppelte an Trockensubstanz auf der Flächeneinheit erzeugt wie ein Kartoffelacker, nämlich 12:6 t/ha Trockensubstanz, und noch eine Ernte von $80 \mathrm{t} / \mathrm{ha}$ Kürbis ist einer Zuckerrübenernte gleichzusetzen. Die Frage des Einsäuerns der wenig haltbaren Kürbisfrüchte ist in gemeinsamer Arbeit mit dem Giessener Institut für Tierernährung gelöst worden. Nach gieriger Aufnahme des Kürbisfutters in frischem und vergorenem Zustand durch Kühe sollen Milch und Milchfett zunehmen.

Während die Bestrebungen zur Züchtung und Einführung der bisher genannten Arten voll anzuerkennen sind oder doch Erfolge für bestimmte Gegenden Mitteleuropas in nicht allzu ferner Zukunft erwarten lassen, fällt diese positive Einstellung bei der Umzüchtung von Hirsearten zu Grünfutterpflanzen bei den meisten Arten schwer. Es ist zuzugeben, dass alle Vertreter der vielen Hirsegattungen eine grössere Dürreverträglichkeit und geringere Saatgutkosten als Vorteile gegenüber Mais für sich buchen können. Dieser verdrängt sie indes nicht nur als Nahrungs- und Kraftfutterpflanze bis ins innerste Afrika hinein, sondern er macht ihnen auch in Europa als Futterpflanze erfolgreich Konkurrenz. Wohl reicht die ökologische 
Streubreite der Hirsen von extrem trockener Steppe bis in die feuchteren Klimate der Subtropen und Tropen. Aber für die meisten Gegenden von Mitteleuropa sind die Hirsen zu frost- und nässeempfindlich, mindestens so wie Mais, sie erleiden durch ungünstige Witterungsperioden Rückschläge, von denen sie sich nur sehr langsam erholen, während ihre extreme Dürreverträglichkeit in Mitteleuropa keine Rolle spielt. Auch enthalten anscheinend alle Formen und Arten der wichtigen Gattung Andropogon das Glykosid Durrhin, das Blausäure entwickelt, und diese hat in den Südstaaten von USA, in Südafrika und Australien bereits öfters zu Erkrankungen des Viehs mit tödlichem Ausgang Veranlassung gegeben. Diese Gefahr wird angesichts der verstärkten Stickstoffdüngung wohl auch in Mitteleuropa akut, wenn Wetterstürze von wüchsigwarmen und sonnenscheinreichen Tagen zu plötzlich kaltem und trübem Wetter auftreten. Anscheinend verbleibt der bei Wuchswetter reichlich aufgenommene mineralische Stickstoff im Zellsaft der Pflanzen, anstatt zu Eiweissbausteinen verarbeitet zu werden.

Immerhin wurden in Giessen Formen der Zuckerhirse, Andropogon oder Sorgum dochna saccharatum $(22,23,24)$, gezüchtet, die im Weinklima von Mitteleuropa mehr Futter als Mais produzierten. Andererseits wurden gerade solche Formen von Mohrenhirse vor wenigen Jahren in Deutschland mit sehr unreeller Reklame und ohne jede vorherige Züchtung angeboten. In ungünstigen Jahren werden die Versprechungen zu Riesengrünfutter nicht Wirklichkeit und so ist die ganze Propaganda öffentlich und gedruckt als Schwindel und Bauernfängerei bezeichnet worden. Es sagt nämlich die Aufnahme der Hirse- wie anderer Arten in das deutsche Artenverzeichnis der landwirtschaftlichen Nutzpflanzen nichts aus über Wert und Bewährung in der Praxis, sondern weist nur auf die Möglichkeit einer züchterischen Bearbeitung hin.

Ein extrem generativer Typ ist die Rispenhirse, Panicum miliaceum. Vielleicht können aus der Population der Art blattreichere Formen ausgelesen werden. Aber der Komparativ ist hier nur relativ und sagt weniger aus als wenn man von blattreichen Formen sprechen könnte. Ob eine Züchtung zu wertvollen Futterpflanzenformen gelingt, muss bezweifelt werden.

In Ungarn wurde am Anfang der 20er Jahre Mohar, Setaria italica, häufiger angebaut, der als besondere Varietät die wissenschaftliche Bezeichnung var. moharia, also in seltener Wortschöpfung ein latinisiertes ungarisches Wort, erhalten hat. Er wird immer wieder als Futterpflanze auch in Mitteleuropa genannt, hat aber in Ungarn bis Ende der 30er Jahre dem Futter- und Silomais Platz machen müssen.

Dieselbe Firma, die mit dem marktschreierischen Angebot für Mohrenhirse auf leichte Art hat Geld verdienen wollen, vertreibt noch eine andere Art, die Futtermalve, Malva crispa, in einer kraus- und in einer glattblättrigen Sorte. Der Futtermalve werden alle Vorzüge nachgerühmt, die eine ideale Futterpflanze haben soll: Billigkeit des Saatgutes, gute Schattengare zur Unterdrückung von Unkraut, grosse Massenwüchsigkeit, hoher Futterwert und ebenso hoher Vorfruchtwert. Aber die Ansprüche der Art an die Bodenreaktion, den Wasserhaushalt und den Nährstoffreichtum des Bodens sind hoch, ebenso die Ansprüche an die Bodentemperatur. Dabei ist die Keimfähigkeit wegen grosser Hartschaligkeit, wenn die Körner nicht mit Hitze behandelt sind, sehr gering, nämlich oft weniger als $20 \%$, 
eine Hackpflege ist wegen langsamer Jugendentwicklung und nach jedem Schnitt notwendig, bei trockener Witterung ist das Wachstum nichts weniger als üppig, wegen Sperrigkeit muss die Futtermalve auch als Grünfutter gehäckselt werden, nicht nur zur Einsäuerung, die Stengel verholzen, nachdem sie sich verzweigt haben, so dass die Nutzungsdauer zu bester Futterqualität kurz ist, nach allerdings williger Aufnahme von gehäckselter Futtermalve durch Milchkühe sinkt die Milchmenge bei immerhin steigendem Fettgehalt, bei Einsäuerung ergeben sich wegen des hohen Wassergehaltes grosse Schwierigkeiten, bei der Heuwerbung übertrocknen und zerbröseln die Blätter, bevor die dicken Stengel dürr geworden sind. Es ist also über die Futtermalve und ihre Züchtung der Stab zu brechen: Auf den von ihr beanspruchten Böden gibt es wahrlich bessere Futterpflanzen.

In dieser Weise könnten noch viele Arten genannt werden, deren Anbau zu Futterzwecken in Mitteleuropa versucht oder propagiert worden ist, z.B. Wintererbse, Pisum sativum arvense hiemale, Comfrey, Symphytum peregrinum, Bienenpflanze oder Büschelschön, Phacelia tanacetifolia, vor allem aber Topinambur, Helianthus tuberosus. Aber sehr viel ist von ihrer züchterischen Bearbeitung und ihrem Anbau, abgesehen von Sonderfällen, nicht zu erhoffen.

In Rückschau auf das Gesagte ist zu betonen, dass die Züchtung neuer Arten zu Kulturpflanzen und die Übernahme von Nutzpflanzen anderer Gebiete in das mitteleuropäische Klima nicht schnell und leicht zu bewerkstelligen ist. Immerhin konnten in neuerer und neuester Zeit in Mitteleuropa Arten als Futterpflanzen zu grösserer Verbreitung gebracht oder ganz neu eingeführt werden, die auch das Interesse der finnischen Landwirtschaft verdienen dürften. Es ist dabei auf die in Mitteleuropa bisher nicht sehr bekannte Verwendung des Hafers als Grünfutterpflanze mit starken und stärksten Stickstoffgaben hinzuweisen, andererseits auf Ölrettich und Sonnenblume, von denen beiden in Giessen neue, erfolgversprechende Sorten gezüchtet worden sind. Auch bei Sommerraps, Wasserrübe und Markstammkohl hat die Züchtung grosse Fortschritte gemacht.

Daneben mussten allerdings sehr viele Arten aufgezählt werden, die nicht in die erste Gruppe, d.h. nicht zu den als wertvoll erprobten Futterpflanzen gehören und wohl auch nicht in die zweite Gruppe der noch zu erprobenden Arten einzureihen sind, sondern die, wie es in der Bibel heisst, als zu leicht befunden worden sind und verworfen werden müssen. Es besteht eben auch auf dem Gebiet des Futterbaues und der Auswahl der Futterpflanzenarten der Bibelspruch zu vollem Recht: Prüfet alles und behaltet das Beste.

\section{I T E R A T U R N A C H W E I S}

(1) Bergt, K. 1963. Hafer als Futterpflanze. Untersuchungen über Stoffaufnahme, Stoffbildung und Stoffwanderung. Z. Acker-u. Pflanzenbau 117; 313-341.

(2) Blatrmann, W. 1961. Die Futtererträge von Hafer bei verschiedenen Schnittzeiten. Landbauforsch. Völkenrode 11: 94-99.

(3) Boguslawski, E. v. 1953. Ölfruchtbau. Handb. Landw. 2: 318-387.

(4) - - \& SchuSter, W. 1957. Untersuchungen über den Einfluss von Saatzeit, Standraum und Schnittzeit auf die Leistungen der Sonnenblume (Helianthus annuus L) als Futterpflanze. Z. Acker-u. Pflanzenbau 104: 371-408. 
(5) Esser, J. 1955. Anbaurichtlinien für den Zwischenfruchtbau. Landw. Verl. G.m.b.H., Hiltrup bei Münster/Westf.

(6) - - 1960. Zur Nutzung der Weidelgräser. Grünland, Beilage Tierzüchter 9: 43-44, 47-48.

(7) - - 1962. Futterkohl als Hauptfrucht. Deutsche Landw. Pr. 85: 128-129.

(8) Frandsen, K. J. 1959. Brassica Leaf- and Stem Forage-Plants. Handb. Pflanzenzücht. 2. Aufl. 4: $525-530$.

(9) Fürste, K. 1959 u. 1961. Anbauerfahrungen mit Alexandrinerklee. Z. Acker- u. Pflanzenbau 107: $317-330,391-404$. 113: 83-92.

(10) Grebenscikov, J. 1950. Zur Kenntnis der Kürbisart, Cucurbita pepo L., nebst einigen Angaben über Olkürbis. Züchter 20: 194-207.

(11) Наскваятн, J. \& Troll, H.-J. 1959. Lupinen als Körnerleguminosen und Futterpflanzen. Handb. Pflanzenzücht 2. Aufl. 4: 1-51.

(12) Hö́nner, R. 1958. Ergebnisse einer Herkunftsprüfung mit Alexandrinerklee. Grünland, Beilage Tierzüchter, 7: 73-76.

(13) - - 1960. Das Verhalten von Alexandrinerklee-Herkünften im Trockenjahr 1959. Grünland, Beilage Tierzüchter, 9: 1-4.

(14) - - \& Wagner, F. 1960. Anbauversuche mit Ölrettich (Raphanus sativus L.). Z. Acker- u. Pflanzenbau 111: 258-278.

(15) Kirsch, W. 1935. Anbau und Verfütterung von Markstammkohl. Mitt. Landw. 50: $505-506$.

(16) Klapp, E. 1950. Das Welsche Weidelgras bei verschiedener Nutzung und Düngung. Neue Mitt. Landw, 45: 513 .

(17) Косн, V. 1955. Feldfutter- und Zwischenfruchtpflanzen. Landw.-Angew. Wiss. Nr. 44.

(18) KöhnLeın, J. \& FEnSE, H. 1953. Leistungsvergleich der bekanntesten Markstammkohlzüchtungen. Z. Acker- u. Pflanzenbau 96: 519-531.

(19) - - 1960. Die Leistungen verschiedener einjähriger Feldfutterpflanzen im Anbau als Haupt-, Zweit- und Zwischenfrucht auf Grundmoräneverwitterung in Schleswig-Holstein. Kieler Milchwirtsch. Forschungsber. 2: 231-250.

(20) Konold, O. 1933/34. Das Ergebnis mehrjähriger Untersuchungen an bisher unbekannten Futterund Gründüngungspflanzen. Pflanzenbau 10: 350-459.

(21) Kürten, P. W. 1963. Anbau, Ernte und Verwertung von Stoppelrüben - eine Literaturübersicht. Wirtschaftseig. Futter 9: 89-98.

(22) LAмpe, E. 1958: Nochmals: Riesen-Grünfutter-Gemenge. Grünland, Beilage Tierzüchter 7: $33-35$.

(23) LechneR, L. 1958. "Riesen-Grünfutter-Gemengen in der Bewährung. Ibid. 7: 12 - 13.

(24) $\rightarrow-1960$. Nochmals: Riesen-Grünfutter-Gemenge. Ibid. 7: 40.

(25) LEHLE, H. 1962. Uber morphologische und physiologische Eigenschaften des Alexandrinerklees. Zur Beurteilung von Anbaufragen und einer züchterischen Bearbeitung. Z. Acker- u. Pflanzenbau 115: 57-80.

(26) Limberg, P. 1959. Der Einfluss der Aussaatzeit auf die Ertragsbildung von Lathyrus sativus im Vergleich mit anderen Leguminosen. Ibid. 109: 141-172.

(27) - - \& ENAYAT, R. 1960. Der Einfluss klimatischer Wachstumsfaktoren auf Entwicklung und Ertragsbildung verschiedener Herkünfte von Trifolium alexandrinum L. Ibid. 111; $116-150$.

(28) LöвE, W. 1872. Landwirtschaftlicher Futterbau mit besonderer Rücksicht auf neue Futterpflanzen und Futtergemenge. Wiegandt u. Hempel, Berlin.

(29) Lucanus, R. 1955. Entwicklungsverlauf und Ertragsbildung von Brassica juncea L. Coss. in Abhängigkeit von Temperatur und Tageslänge. Diss. Giessen.

(30) Мгске, А. 1958. Mutationszüchtung beim weissen Steinklee (Melilotus albus) mit Hilfe von Röntgenstrahlen. Z. Pflanzenzücht. 39:419-437.

(31) Müнling, H.-G. 1962/63. Zur Stoffbildung und Stoffspeicherung bei Mais. Z. Acker- u. Pflanzenbau 116: $129-153$.

(32) Primost, E. 1961/62. Ertragsleistung und Futterqualität des Markstammkohls in Abhängigkeit von der Stickstoffdüngung und Anbaumethode. Ibid. 114: 199-216.

(33) Rudorf, W. 1961. Die Sonnenblume, Helianthus annuus L. Handb. Pflanzenzücht. 2. Aufl. 5: $89-114$. 
(34) SAchs, E. 1958. Die Leistung des Alexandrinerklees bei verschiedener Schnittnutzung. Grünland, Beilage Tierzüchter 7:24-25.

(35) Scharrer, K., Schreiber, R. \& Kühn, H. 1955. Über den Futterwert von "Giessener Ölrettich, Sareptasenf „Stamm 40/44, und „Giessener bunte Platterbse». Z. Tierernähr. u. Futtermittelkunde 10: $134-146$.

(36) —- 1960. Uber die Silierfähigkeit und den Futterwert von Ölkürbis (Cucurbita pepo). Ibid. 15: $265-273$.

(37) Scheibe, A. \& Hülsmann, G. 1958. Mutationsauslösung durch Chemikalien beim Steinklee (Melilotus albus). Z. Pflanzenzücht. 39: 299-324.

(38) Schuster, W. 1961. Mehrfaktorielle Versuche zur Anbautechnik beim Silomais. Z. Acker- u. Pflanzenbau 113: 243-262.

(39) Srmon, U. 1957. Weitere Versuche mit Alexandrinerklee, Italienischem Rotklee und deren Gemengen mit Welschem Weidelgras. Bayer. Landw. Jb. 34: 609-616.

(40) SтÄнцгn, A. 1959. Zur Unterscheidung von Alexandriner und Sperrigem Klee (Trifolium alexandrinum L. und T. squarrosum L.). Grünland, Beilage Tierzüchter 8: 6-8.

(41) Ullmann, W. 1957 u. 1958. Uber den Alexandriner- oder ägyptischen Klee, Trifolium alexandrinum L.: Bezeichnungen, Abstammung und Verbreitung, Geschichtliches, Anbauversuche und Anbauausweitung in Vergangenheit und Gegenwart, ferner botanische Uberschau. Saatgutwirtschaft $9: 262-265,293-295,324-325$. 10: $9-11$.

(42) Vömel, A. 1955. Wachstumsverlauf, Entwicklung und Kornertragsbildung der Weissen Lupine (Lupinus albus L.) in Abhängigkeit von klimatischen Faktoren. Z. Pflanzenzücht. 35: $199-238$.

(43) WACKeR, H. 1963. Stoppelrüben im Zwischenfruchtbau (Anbau-Einsäuerung-Fütterung). Landw. Kammer Weser-Ems, H. 10 Oldenburg.

(44) Whitaker, T. W. 1962. Breeding Squash and Pumpkins. Handb. Pflanzenzücht. 2. Aufl. 6: $331-350$.

(45) ZÛRN, F. 1963. Anbau von Hafer zur Grünnutzung. Wirtschaftseig. Futter 9: 40-47.

\title{
SELOSTUS:
}

\section{UUSIA REHUKASVEJA SAKSAN REHUNTUOTANNOSSA}

\author{
A. Stählin
}

Justus Liebig-Yliopisto, Giessen, Saksa

Rehuksi viljeltävien kasvien lajikoostumus on melkoisesti muuttunut Saksassa sodan jälkeen. Toisaalta on kokeiltu ja otettu käyttöön lukuisia kokonaan uusia lajeja, toisaalta on moni jo aikaisemmin Saksassa viljelty laji saanut uutta merkitystä, kun on kehitetty uusia, oloihin paremmin soveltuvia jalosteita ja viljely- ja korjuumenetelmät ovat kehittyneet. Niinpä esim. maissi, jota on Saksassa viljelty jo toistasataa vuotta, on saavuttanut huomattavan viljelylaajuuden etenkin vihantarehukasvina (vuosittain n. $40000-50000$ ha) sen jälkeen kun on ryhdytty viljelemään amerikkalaisia lajikkeita ja vastikään jalostettuja eurooppalaisia hybridejä. Korjuun koneistuminen on myös edistänyt maissin tuotantoa. Ilmastollisesti epäsuotuisilla alueilla ja happamilla mailla viljellään maissin sijasta kauraa, jonka viljely kypsäksi rehuviljaksi on nopeasti taantunut, mutta vihantarehukasvina voimakkaasti lisääntymässä. Kaikki kauralajikkeet, lukuunottamatta kaikkein lyhytkortisimpia ja vähälehtisimpiä leikkuupuintikauroja, soveltuvat tähän tarkoitukseen. Uusia lehteviä jalosteita on saatu italialaisesta raiheinästä ja kevätrapsista, ja myös mustasinappia (Brassica nigra) jalostetaan rehuominaisuuksia silmälläpitäen. Oljyretikka (Raphanus sativus oleiformis) on saanut vankan jalansijan Saksan rehunviljelyssä, koska lajin verraten alhainen sinappiöljypitoisuus Sinapis- ja Brassica-lajeihin verrattuna on eduksi sekä karjan terveydelle että maidontuotannolle. Giessenissä on onnistuttu jalostuksella parantamaan öljyretikan laonkestävyyttä. 
Jo vanhastaan harjoitettu rehunauriinviljely on saamassa entistä tärkeämmän merkityksen uusien lehtevien jalosteiden ansiosta. Korjuumenetelmissä tapahtunut kehitys on lisännyt nauriin viljelyä säilörehuksi. Myös rehukaalin jalostus on tuottanut hyviä tuloksia.

Aivan uusi rehukasvilaji, joka sodan jälkeen on saavuttanut Saksassa melkoisen viljelylaajuuden, on Aleksandrian apila (Trifolium alexandrinum), jota viljellään esim. seoksina italialaisen raiheinän kanssa. Kokeiltavina ja jalostettavina Giessenissä ovat myös Persian apila (Trifolium resupinatum), josta odotetaan kilpailijaa Aleksandrian apilalle, valkomesikkä (Melilotus albus), jonka rehuominaisuuksia pyritään parantamaan, sekä useat Lupinus- ja Lathyrus-lajit. Erilaiset hirssilajit ovat osoittautuneet niin paljon lämpöă vaativiksi, että niitä voidaan viljellä vain kaikkein lämpimimmillä seuduilla. Samoin on laita öljykurpitsan (Cucurbita pepo styriaca), joka viihtyy viininviljelyalueilla. Siitä samoin kuin auringonkukasta (Helianthus annuus) on Giessenissä myös kehitetty uusia lajikkeita. 